\title{
MRS Invites Nominations for the Von Hippel Award, MRS Medal, and Turmbull Lectureship
}

The Materials Research Society is seeking nominations for the Von Hippel Award, the MRS Medal, and the Turnbull Lectureship. The deadline for nominations is June 1, 1996. These awards will be presented at the 1996 MRS Fall Meeting, December 2-6, in Boston.

The MRS Awards Program recognizes outstanding contributors to the progress of materials research. Nomination forms and details about eligibility and nomination criteria are available from Anne Wagner, Materials Research Society, 9800 McKnight Road, Pittsburgh, PA 152376006; phone 412-367-3004 ext. 102; fax 412-367-4373. Further information about each award can also be obtained from MRS homepage on the World Wide Web: http://www.mrs.org.

\section{Von Hippel Award Acknowledges Outstanding Interdisciplinary Work in Materials Research}

The Von Hippel Award, first presented to Arthur R. von Hippel whose interdisciplinary and pioneering research typified the spirit of the award, is the Materials Research Society's highest honor. The recipient is recognized for brilliance and originality combined with vision that transcends the boundaries of conventional scientific disciplines. The Award includes a $\$ 7,500$ cash prize, honorary membership to MRS, and a unique trophy-a mounted ruby laser crystal symbolizing the manyfaceted nature of materials research.

Selection of the recipient is determined by a vote of the MRS Council. The recipient will be invited to speak at the Awards Ceremony.

\section{Turnbull Lectureship Honors Career of an Outstanding} Researcher and Communicator

The David Turnbull Lectureship recognizes the career of a scientist who has made outstanding contributions to understanding materials phenomena and properties through research, writing, and lecturing, as exemplified by the life work of David Turnbull. While honoring the accomplishments of the recipient, the Turnbull
Lectureship is intended to support and enrich the materials research community.

The recipient will give a technical lecture of broad appeal at a designated session of the 1996 MRS Fall Meeting. The Turnbull Lecturer will receive a $\$ 3,000$ honorarium and a citation plaque, along with travel expenses paid to enable the recipient to address MRS Sections and University Chapters, and/or participate in the production of a video version of the lecture.

\section{MRS Medals Recognize Recent Discoveries or Advancements in Materials Science}

The MRS Medals offer public and professional recognition of exceptional recent achievements in materials research. Two medals will be awarded for specific outstanding recent discoveries or advancements which are expected to have major impacts on the progress of any materialsrelated field.

Each award consists of a $\$ 2,000$ cash prize, an engraved and mounted medal, and a citation certificate.

MIEIS

\section{Previous Recipients of the MRS Medal}

Arthur J. Freeman

Northwestern University

Duward F. Shriver

Northwestern University

Bernard S. Meyerson

IBM T.J. Watson Research Center

Shigeyuki Sõmiya

Nishi Tokyo University

L. Eric Cross

The Pennsylvania State University

Stephen J. Pennycook

Oak Ridge National Laboratory

Wolfgang Krätschmer

Max-Planck Institute für Kernphysik

Donald R. Huffman

University of Arizona

Max G. Lagally

University of Wisconsin-Madison

Kenneth S. Suslick

University of Illinois at

Urbana-Champaign

Federico Capasso

ATET Bell Laboratories

Rudolf M. Tromp

IBM T.J. Watson Research Center 


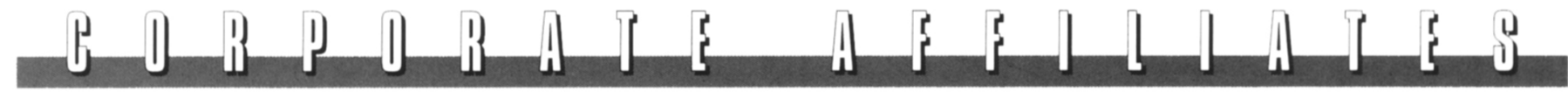

(As of January 1996)

\section{Materials Research Society would like to thank the following for their financial support:}

ABB Extrel Corporation

Advanced Control Systems Corp.

Advanced Energy Industries, Inc.

Advanced Micro Devices, Inc.

Advanced Photovoltaic Systems, Inc.

AEA Technology

Aerospace Corp.

Aetrium Sienna Technologies

AG Associates

Air Products and Chemicals, Inc.

AIXTRON, Inc.

AKZO Nobel Chemicals, Inc.

Aldrich Chemical Company

Allied-Signal, Inc./Advanced

Microelectronic Materials

Allied-Signal, Inc./Research \& Technology

Aluminum Company of America

Aluminum Research Board

American Chemical Society

American Vacuum Process

American Xtal Technology

AMRAY, Inc.

Anatech, Ltd.

APD Cryogenics, Inc

Applied Materials

Argonne National Laboratory

Asahi Glass Co., Ltd

AST elektronik GmbH

ASTeX

AT\&T Bell Laboratories

ATRIX Laboratories, Inc.

Automotive Composites Consortium

Balzers

Battelle Pacific Northwest Labs

Bayer Corp.

Bellcore

BIOSYM/Molecular Simulations

Blake Industries, Inc.

British Nuclear Fuels

Brookhaven Instruments Corp.

Brookhaven National Laboratory

Cabot Corp.

Cameca Instruments, Inc.

Canon, Inc.

CAPE Simulations

Centre European pour le Recherche

Nucleaire (CERN)

CFM Technologies, Inc.

Charles Evans \& Associates

Chemat Technology, Inc.

China Technical Consultants, Inc.

Chisso Corp.

CNRS

Cober Electronics

Coherent Laser Group

Commonwealth Scientific Corp.

Communications and Power Industries, Inc.

Comstock Inc.

Conductus, Inc.

Consortium für Elektrochemische Ind. GmbH

Corning, Inc.

Courtaulds

CREE Research, Inc.

Creekside Technologies Corp.

Current Science Group

CVC Products, Inc.

Cymer Laser Technologies

Dainippon Screen Mfg. Co., Ltd.

DCA Instruments, Inc.

Denton Vacuum, Inc.

Digital Equipment Corp

Digital Instruments, Inc.

Dow Chemical Co

Dow Corning Corp.

DSM Research

Dynatek Dalta Scientific Instruments

Dytech Internationa

Eastman Kodak Company

Eaton Corp.

EG\&G Nuclear Instruments

E.l. duPont de Nemours \& Co. Ino

Electric Power Research Institute (EPRI)

Electricité de France

Elsevier Science Publishing Co., Inc.

EMCORE Corp.

Energy Conversion Devices
Engelhard Corp.

Enrat-Nonius Co.

EPI/MBE Products Group

EPRI

ES Microware

Evans East

Exxon Production Research Co.

Exxon Research and Engineering $\mathrm{Co}$.

Federal Highway Administration

FEI Co.

E.A. Fischione Instruments, Inc.

Fisons Instruments

Ford Motor $\mathrm{Co}$.

FSI International

Fuji Electric Co., Ltd

Fujikin of America, Inc

Gas Research Institute

Gatan, Inc.

Gelest, Inc.

Genentech, Inc.

General Electric $\mathrm{Co}$

General Motors Research

Genus, Inc.

Golden Technologies

Goodfellow Corp.

Gordon \& Breach Publishers, Inc.

Gould Electronics, Inc

Granville-Phillips $\mathrm{Co}$.

Groupe de Dynamique des Phase Condenses

Haldor Topsøe A/S

Hauzo Techno Coating Europe

Hewlett-Packard Co.

High Voltage Engineering Europa B.V

Hitachi, Ltd.

Hitachi Scientific Instruments

Hoechst AG

Hoechst Celanese Research Division

Howmedica

Hughes Research Laboratories

Huntington Mechanical Laboratories

Hysitron, Inc.

IBM AdStaR

BM Analytical Services

IBM Corp.

IHI Research

Inorgtech

Institut für Schicht und Ionentechnik (ISI)

Institute for Scientific Information (ISI)

Institute of Physics Publishing (IOP)

Instron Corp.

Instruments SA, Inc

instruments SA, Inc./Riber Division

Intel Corp.

International Center for Materials

Research (ICMR)

Ion Tech, Inc.

ITAC, Ltd

JCPDS-International Centre for Diffraction Data

JEOL USA, InC.

Johnsen Ultrava

Johnson Controls, Inc.

Johnson \& Johnson

Johnson \& Johnson Protessional

k-Space Associates, Inc.

Kaneka Corp.

Kawasaki Steel Corp.

Keithley Instruments, Inc.

Kimball Physics, Inc.

Kobe Steel USA, Inc.

Komag, Inc.

Kratos Analytica!, Inc

Kurt J. Lesker Co.

Kyocera Corp.

Lafarge Fondu Internationa

Lake Shore Cryotronics, Inc.

Lambda Physik, Inc.

Lawrence Berkeley Laboratory

Lawrence Livermore National Laboratory

Lawrence Semiconductor Research

Laboratory

Legacy Systems, Inc.

ockheed Idaho Technologies Co.

Lockheed Martin Energy Systems

Los Alamos National Laboratory

Lumonics, Inc.

Magnet Sales \& Manufacturing Co.
Marcel Dekker, Inc

Materials and Technologies Corp.

Materials Research Corp.

Materials Research Group

Mattson Technology

MCP Wafer Technology, Ltd.

MDC Vacuum Products Corp.

Medisorb Technology International

MEL Chemicals

MEMC Electronic Materials

MER Corp.

Microwave Materials Technologies, Inc.

Millipore Corp.

Mitsubishi Electric Semiconductor Laboratories

Mitsubishi Materials Corp.

Mitsui Engineering \& Shipbuilding Co., Ltd.

Mitsui Toatsu Chemicals, Inc

MKS Instruments, Inc.

Morton Int'l Advanced Materials

Motorola APRDL

MR Semicon, Inc.

MVSystems, Inc.

n\&k Technology

Nano Instruments, Inc.

Nanophase Technologies

Nanotec Corp.

NASA Lewis Research Center

National Electrostatics Corp.

National Renewable Energy Laboratory (NREL)

National Semiconductor

NEC Corp.

NEC Research Institute, Inc.

Neocera, Inc.

New Focus, Inc.

Niki Glass Co.

Nikko Hitech International, Inc.

Nippon Oil Corp.

Nippon Steel Corp.

Nissei Sangyo America, Ltd.

Nor-Cal Products, Inc.

Northern Telecom, Ltd.

Novapure Corp.

NOVOCONTROL

Nuclear Regulatory Commission

Oak Ridge National Laboratory

Omicron Associates

OnTrak Systems, Inc.

OPTOVAC

Oxford Applied Research

Oxford Instruments, inc

Park Scientific Instruments

Paterson Instruments PTY, Ltd

Perseptive Biosystems, Inc.

Philips Electronic Instruments Co. (PEI)

Physical Electronics

Plasma Sciences, Inc.

Plasma-Therm, Inc.

Plenum Publishing $\mathrm{Co}$.

Portland Cement Association

Precision Manufacturing Center

President Enterprises Co.

Princeton Gamma-Tech, Inc.

Pryor Knowledge Systems, Inc.

Pure Tech, Inc.

Quantum Design, Inc

Read-Rite Corp.

Research and PVD Materials Corp.

Rexham Custom

RHK Technology

Rigaku/USA, Inc.

RIMCOF

Rockwell International Corp.

ROITECH

Sandia National Laboratories

Santa Clara Plastics

Sanyo Electric Co. Ltd.

Schlumberger Doll Research

Schumacher/Air Products \& Chemicals Unit

Seagate Technology, Inc

Sematech, Inc.

Semiconductor Processing $\mathrm{Co}$.

Sensarray Corp

SGS-Thomson Microelectronics

Sharp Corp.

Siemens Analytical X-Ray Instruments, Inc.

Siltec Silicon

Smith \& Nephew Research, Ltd

Solarex Corp.

Solartron Instruments

Solid State Equipment Co.

Sony Co., Ltd.

Sophisticated Alloys, Inc.

SOPRA, Inc.

South Bay Technology, Inc.

Southwest Research institute

Spectra-Physics Lasers, inc.

Spire Corp.

Springer-Verlag New York, Inc.

Staib Instrumente $\mathrm{GmbH}$

Strem Chemicals, Inc.

SubMicron Systems, Inc.

Sumitomo Electric USA, Inc.

Sumitomo Sitix Corporation

SUNY-Albany

Superior Vacuum Technology, Inc

Surface/Interface, Inc.

SVT Associates

TDA Research

Telemark

Tencor Instruments

Texas Instruments, Inc.

Thermionics Laboratory, Inc

3M Fiber Optics

Tokyo Instruments, Inc.

Topcon Technologies, Inc.

TopoMetrix Corp. 